\title{
Role of Socioeconomic Status in Hypertension among Chinese Middle-Aged and Elderly Individuals
}

\author{
Xinyi Wu $(\mathbb{C}$ and Zhonghua Wang $(\mathbb{D}$ \\ Department of Health Economics \& Management, School of Health Policy \& Management, Nanjing Medical University, \\ Nanjing 211100, China \\ Correspondence should be addressed to Zhonghua Wang; wzh04@126.com
}

Received 6 May 2019; Accepted 3 September 2019; Published 13 October 2019

Academic Editor: Salvatore Corrao

Copyright (c) 2019 Xinyi Wu and Zhonghua Wang. This is an open access article distributed under the Creative Commons Attribution License, which permits unrestricted use, distribution, and reproduction in any medium, provided the original work is properly cited.

\begin{abstract}
Hypertension is an important global health concern. The relationship between hypertension and socioeconomic status (SES) has been extensively studied. However, the role of SES in hypertension is still controversial, and this kind of study is sorely lacking among Chinese middle-aged and elderly individuals. The data of this study come from the China Health and Retirement Longitudinal Survey (CHARLS) released in May 2017. A total of 21,126 people from all around China, with ages older than 45 years, were enrolled in the questionnaire survey. Hypertension was determined according to the entry in CHARLS ("Do you have doctor-diagnosed hypertension?"), and 17,676 people responded to this entry. The basic demographic and SES information were collected. Multivariate logistic regression was used to evaluate the risk factors of hypertension. Concentration index was used to measure inequality of hypertension incidence. Among the investigated middle-aged and elderly individuals, 5,177/17,676 (29.3\%) had doctor-diagnosed hypertension. Multivariate logistic regression implied that individuals older than 55 years (OR 1.436, 95\% CI 1.085-1.900 for age interval of 55-64 years; OR 2.032, 95\% CI 1.455-2.839 for age interval of 65-74 years; OR 1.672, 95\% CI 1.031-2.714 for age interval of older than 75 years), male (OR 0.038, 95\% CI 0.595-0.986), overweight (OR 2.47, 95\% CI 1.462-4.183), and diabetes (OR 3.159, 95\% CI 2.129-4.687) were associated with hypertension. For society support, individuals in the lowest quintile were more likely to suffer hypertension. Concentration index results suggested that different income groups did not show inequality on hypertension incidence. Elder age, male, overweight, diabetes, and poor society support were suggested to be associated with hypertension incidence among middle-aged and elderly individuals in China. Our study provides implications for controlling and managing hypertension.
\end{abstract}

\section{Introduction}

Hypertension is a big issue of global health and poses a great challenge for public health worldwide. It has been recognized as an important risk factor for cardiovascular diseases (CVD) $[1,2]$. Globally, hypertension attributes to about $50 \%$ CVD mortality. Effective control of blood pressure is valuable for preventing the incidence of CVD [3].

In China, the morbidity of hypertension had been apparently rising in the past semicentury. Since 1958, four nation-wide investigations were conducted in 1958, 1979, 1991, and 2002, and the results showed that the hypertension prevalence rate was rising from $5-11 \%$ to $24-27 \%$ [4]. It was estimated in 2010 that approximately 200 million people suffered from hypertension in China [5].

In the Chinese guidelines for the management of hypertension (version 2010) [5], which is also a guideline for hypertension diagnosis used by Chinese doctors, hypertension was defined as follows: in the absence of antihypertensive drugs, blood pressure is measured at least 3 times in different days, with a systolic blood pressure $\geq 140 \mathrm{mmHg}$ and/or diastolic blood pressure $\geq 90 \mathrm{mmHg}$. Individuals who had been diagnosed as having hypertension by a doctor or used antihypertensive drugs are also defined as hypertension. In 2017, at the annual meeting of the American Heart Association (AHA), a new guideline for hypertension was 
proposed, redefining hypertension as blood pressure $\geq 130$ / $80 \mathrm{mmHg}$ [6]. However, it was also noted that this document was for guidance only, and the decision made by the clinician according to the specific situation of the patient is the most important. It reflects that hypertension is getting more and more attention and highly valued [6].

As a special part of the population, health care of middleaged and elderly individuals always occupies an important status in the public health services system [7, 8]. Hypertension and the consequent CVD in middle-aged and elderly individuals bring a huge burden for public health [1]. Moreover, the aging problem in China is getting more and more serious. It was reported that, in 2010, there were 111 million people aged $65+$ years and 19.3 million aged $80+$ years, and it was estimated that the number will increase to 400 and 150 million, respectively, by $2050[9,10]$. In the near future, China will be one of the countries with most serious aging problem [10].

It had been indicated in many researches that socioeconomic status (SES) was closely related to hypertension. However, different researches presented some conflicting results [11-14]. There are still very limited literatures discussing the relationship between SES and hypertension prevalence in low- and middle-income countries, especially China $[4,11]$. China Health and Retirement Longitudinal Study (CHARLS) is a national representative longitudinal survey of the middle-aged and elderly individuals in China. Items in this survey include health circumstance, and social and economic status of persons from across the country, both urban and rural regions, aging 45 years or older $[4,11]$. CHARLS provides high-quality database for studying the relationship between SES and hypertension among middleaged and elderly individuals in China.

\section{Methods}

2.1. Data Source. The data used for this study came from the CHARLS database [11]. CHARLS is hosted by the National School of Development of Peking University, and performed by the Institute of Social Science Survey and Youth league committee of Peking University. The aim of the survey was to collect high-quality microdata of Chinese families and individuals older than 45 years. The results can be used to analyze the aging problem of China, promote the interdisciplinary research of the aging problem, and provide scientific basis to formulate and improve relevant policies in China. The national baseline survey of CHARLS started in 2011 and was tracked every two years. One year after the survey ended, the data were freely available to the academic community. CHARLS was performed in 2011, 2013, 2104 ("Life History Survey" special project), and 2015, covering 23 thousand interviewees of 12.2 billion families from both urban and rural regions in 28 provinces/municipalities/ autonomous regions by 2015. Items in the CHARLS questionnaire mainly include basic personal information, family structure, health status, physical measurement, medical service utilization, medical insurance, assets, basic community conditions, etc. The data in this study were followed up in 2015 and released publicly in May 2017.
2.2. Definitions. Hypertension was determined according to the entry in CHARLS ("Do you have doctor-diagnosed hypertension?"). Doctors diagnosed hypertension based on the Chinese guidelines for the management of hypertension (version 2010) [5], in which hypertension is defined as follows: in the absence of antihypertensive drugs, blood pressure is measured at least 3 times on different days, with a systolic blood pressure $\geq 140 \mathrm{mmHg}$ and/or diastolic blood pressure $\geq 90 \mathrm{mmHg}$. Individuals who had been diagnosed as having hypertension by a doctor or used antihypertensive drugs are also defined as hypertension.

Socioeconomic status (SES) is a comprehensive indicator measuring the social status of individuals and can reflect the level of social and economic status of members in the society. Income, education, and occupation, which represent different aspects of social and economic status, are regarded as three major indicators of SES [14, 15].

Concentration index (CI) is a health equity index in health economics and is used to measure health inequities associated with SES. The CI value is between -1 and 1 . A positive value of CI indicates that the prevalence of hypertension tends to be among the high-income people, and a larger value indicates a greater inclination to high-income people, and vice versa for a negative CI value. A CI value of 0 indicates that people in different income groups are equally likely to have hypertension [16].

2.3. Research Variables. The outcome variable is hypertension, which is defined as described earlier. SES factors are divided into four aspects: demographic factors, economic factors, health and health behavior, and psychosocial factors (Table S1). The demographic factors include gender, age (divided into four degrees: 45-54, 55-64, 65-74, and older than 75 years) and residence (urban or rural). The economic factors include educational level, income (averaged into 5 levels), working status, and medical insurance. Health and health behavior include BMI index, exercise intensity, smoking, and diabetes. BMI value was calculated by dividing weight in kilograms by height in meters squared, and the classification criteria are as follows: underweight, <18.5; normal, 18.5-23.9; and overweight, $\geq 24$. The psychosocial factors include depression evaluation, social support (averaged into 5 levels), and social participation (whether participating in social activities).

2.4. Statistical Analysis. IBM SPSS Statistics 21.0 (IBM, Chicago, IL, USA) was used for statistical analysis. Multiple imputation was used to deal with the missing data. We selected factors that were significantly associated with hypertension in the univariate analysis $(P<0.20)$. Candidate variables were entered into stepwise multivariate logistic regression using a backward selection model. To better assess the association between various SES factors and hypertension prevalence, bootstrapping (10,000 resampling) analysis was used to estimate the odds ratios (OR) and 95\% confidence intervals (CI). $P<0.05$ was considered to be statistically significant. The inequity analysis of hypertension prevalence was evaluated by using the concentration index. 


\section{Results}

A total of 21,126 Chinese middle-aged and elderly (45 years or older) individuals were enrolled into the questionnaire survey of CHARLS. The characteristics of demographic factors, economic factors, health and health behavior, and psychosocial factors of these people are summarized in Table 1. Among these people, 17,676 responded to the question about hypertension, and 5,177/17,676 (29.3\%) had doctor-diagnosed hypertension.

The results of evaluation of the association between various SES factors and hypertension prevalence using multivariate logistic regression are shown in Table 2. Several factors were found likely to be risk factors of hypertension. Males were more likely to suffer hypertension than females based on the CHARLS results $(P=0.038$, OR $0.038,95 \%$ CI 0.595-0.986). Age is another important factor associated with hypertension prevalence. Compared with the youngest group of 45-54 years, the other three age groups are significantly more likely to have hypertension $(P<0.05)$, especially the 65-74 years old group, with an OR value of 2.032 and $95 \%$ CI value of $1.455-2.839$. The 55-64 and older than 75 years groups presented OR values of 1.436 and 1.672, and $95 \%$ CI values of $1.085-1.900$ and $1.031-2.714$, respectively. Besides, consistent with most other studies investigating risk factors of hypertension and CVD, overweight and diabetes were significantly associated with hypertension $(P<0.05)$; the OR values were as high as 2.473 and 3.159 , with $95 \%$ CI values of $1.462-4.183$ and 2.129-4.687, respectively. Moreover, social support was also found to be associated with hypertension prevalence. People with better social support tended to stay away from hypertension. Compared with individuals receiving lowest social support, people with lower support (OR 0.618, 95\% CI 0.449-0.851), medium support (OR $0.625,95 \%$ CI 0.417-0.936), and highest support (OR $0.608,95 \%$ CI $0.432-0.856$ ) all showed significantly lower hypertension prevalence $(P<0.05)$. We did not find other SES factors that were associated with the prevalence of hypertension in these Chinese individuals.

At last, concentration index was used to evaluate the equity of the prevalence of hypertension in different income groups. The results showed that individuals in all income groups, no matter whether men or women, were equally likely to suffer hypertension in China (Table 3 ).

\section{Discussion}

Hypertension poses a sever challenge to public health and had been regarded as a potential risk for CVD $[1,3]$. To better control the prevalence of hypertension, investigating associated risk factors is of great significance. It had been implied in many researches that some aspects of SES are closely associated with hypertension prevalence. However, some results of this issue are controversial, and more studies need to be conducted in low- and middle-income countries, especially China $[11-15,17]$.

The most recent nation-wide survey on hypertension prevalence in China was conducted in 2002, and the
TABLE 1: Characteristics of the investigated population.

\begin{tabular}{|c|c|}
\hline Item & Frequency $(n(\%))$ \\
\hline \multicolumn{2}{|l|}{ Demographic factors } \\
\hline \multicolumn{2}{|l|}{ Gender } \\
\hline Male & $10148(48.0)$ \\
\hline Female & $10978(52.0)$ \\
\hline \multicolumn{2}{|l|}{ Age (years) } \\
\hline $45 \sim$ & $4410(29.3)$ \\
\hline $55 \sim$ & $5874(39.0)$ \\
\hline $65 \sim$ & $3209(21.3)$ \\
\hline $75 \sim$ & $1553(10.3)$ \\
\hline \multicolumn{2}{|l|}{ Residence } \\
\hline Urban & $4353(23.5)$ \\
\hline Rural & $14189(76.5)$ \\
\hline \multicolumn{2}{|l|}{ Economic factors } \\
\hline \multicolumn{2}{|l|}{ Education level } \\
\hline Illiteracy & $9178(43.5)$ \\
\hline Primary school & $4568(21.7)$ \\
\hline Junior high school & $4474(21.2)$ \\
\hline Senior high school & $2343(11.1)$ \\
\hline University or higher & $521(2.5)$ \\
\hline \multicolumn{2}{|l|}{ Income } \\
\hline Lowest & $2153(20.1)$ \\
\hline Lower & $2140(19.9)$ \\
\hline Medium & $2153(20.1)$ \\
\hline Higher & $2141(19.9)$ \\
\hline Highest & $2145(20.0)$ \\
\hline \multicolumn{2}{|l|}{ Working status } \\
\hline Working & $11847(65.5)$ \\
\hline Retirement & $5915(32.7)$ \\
\hline Unemployment & $312(1.8)$ \\
\hline \multicolumn{2}{|l|}{ Medical insurance } \\
\hline No & $861(4.7)$ \\
\hline Yes & $17414(95.3)$ \\
\hline \multicolumn{2}{|l|}{ Health and health behavior } \\
\hline \multicolumn{2}{|l|}{ BMI } \\
\hline Underweight & $749(5.8)$ \\
\hline Normal & $4915(37.9)$ \\
\hline Overweight & $7300(56.3)$ \\
\hline \multicolumn{2}{|l|}{ Diabetes } \\
\hline No & $16154(92.2)$ \\
\hline Yes & $1376(7.8)$ \\
\hline \multicolumn{2}{|l|}{ Exercise scoring } \\
\hline Low intensity & $2955(49.6)$ \\
\hline Medium & $991(16.6)$ \\
\hline High intensity & $2013(33.8)$ \\
\hline \multicolumn{2}{|l|}{ Smoking } \\
\hline No & $11969(80.1)$ \\
\hline Yes & $2968(19.9)$ \\
\hline \multicolumn{2}{|l|}{ Psychosocial factors } \\
\hline \multicolumn{2}{|l|}{ Depression } \\
\hline No & $12088(73.0)$ \\
\hline Yes & $4473(21.2)$ \\
\hline \multicolumn{2}{|l|}{ Social support } \\
\hline Lowest & $2898(20.1)$ \\
\hline Lower & $4113(28.5)$ \\
\hline Medium & $1763(12.2)$ \\
\hline Higher & $2782(19.3)$ \\
\hline Highest & $2888(20.0)$ \\
\hline \multicolumn{2}{|l|}{ Social participation } \\
\hline No & $7852(37.2)$ \\
\hline Yes & $8967(42.4)$ \\
\hline
\end{tabular}


TABLE 1: Continued.

\begin{tabular}{cc}
\hline Item & Frequency $(n(\%))$ \\
\hline Hypertension & \\
No & $12499(70.7)$ \\
Yes & $5177(29.3)$ \\
\hline
\end{tabular}

TABLE 2: Logistic regression analyses of factors affecting hypertension prevalence.

\begin{tabular}{|c|c|c|c|}
\hline Factors & $\mathrm{OR}^{*}$ & $95 \% \mathrm{CI}^{*}$ & $\begin{array}{c}P \\
\text { value* }^{*}\end{array}$ \\
\hline \multicolumn{4}{|l|}{ Age (control, 45 ) (years) } \\
\hline $55 \sim$ & 1.436 & $1.085-1.900$ & 0.011 \\
\hline $65 \sim$ & 2.032 & $1.455-2.839$ & 0.000 \\
\hline $75 \sim$ & 1.672 & $1.031-2.714$ & 0.037 \\
\hline Gender (control, male) & 0.765 & $0.595-0.986$ & 0.038 \\
\hline Residence (control, urban) & 0.851 & $0.609-1.190$ & 0.345 \\
\hline \multicolumn{4}{|c|}{ Education level (control, illiteracy) } \\
\hline Primary school & 0.911 & $0.685-1.213$ & 0.525 \\
\hline Junior high school & 0.745 & $0.543-1.022$ & 0.068 \\
\hline Senior high school & 0.701 & $0.442-1.112$ & 0.131 \\
\hline University or higher & 0.760 & $0.292-1.982$ & 0.575 \\
\hline \multicolumn{4}{|l|}{ Income (control, lowest) } \\
\hline Lower & 1.264 & $0.912-1.750$ & 0.159 \\
\hline Medium & 0.926 & $0.660-1.299$ & 0.656 \\
\hline Higher & 0.759 & $0.530-1.086$ & 0.131 \\
\hline Highest & 1.039 & $0.709-1.524$ & 0.844 \\
\hline \multicolumn{4}{|c|}{ Working status (control, working) } \\
\hline Retirement & 0.516 & $0.260-1.025$ & 0.059 \\
\hline Unemployment & 0.667 & $0.334-1.334$ & 0.252 \\
\hline \multicolumn{4}{|c|}{ Medical insurance (control, no) } \\
\hline & 0.771 & $0.446-1.331$ & 0.351 \\
\hline \multicolumn{4}{|l|}{ BMI (control, normal) } \\
\hline Underweight & 0.960 & $0.563-1.638$ & 0.882 \\
\hline Overweight & 2.473 & $1.462-4.183$ & 0.001 \\
\hline \multicolumn{4}{|l|}{ Diabetes (control, no) } \\
\hline & 3.159 & $2.129-4.687$ & 0.000 \\
\hline \multicolumn{4}{|l|}{$\begin{array}{l}\text { Exercise scoring (control, low } \\
\text { intensity) }\end{array}$} \\
\hline Medium & 0.775 & $0.568-1.057$ & 0.107 \\
\hline High intensity & 0.810 & $0.634-1.037$ & 0.094 \\
\hline Smoking (control, no) & 0.810 & $0.579-1.133$ & 0.218 \\
\hline Depression (control, no) & 1.231 & $0.971-1.561$ & 0.086 \\
\hline \multicolumn{4}{|c|}{ Social support (control, lowest) } \\
\hline Lower & 0.618 & $0.449-0.851$ & 0.003 \\
\hline Medium & 0.625 & $0.417-0.936$ & 0.023 \\
\hline Higher & 0.733 & $0.516-1.041$ & 0.082 \\
\hline Highest & 0.608 & $0.432-0.856$ & 0.004 \\
\hline \multicolumn{4}{|c|}{ Social participation (control, no) } \\
\hline & 1.191 & $0.955-1.486$ & 0.120 \\
\hline
\end{tabular}

${ }^{*}$ Significant differences are in bold.

prevalence rate was $24-27 \%$ [4]. In this study, we found that 5,177/17,676 (29.3\%) people had doctor-diagnosed hypertension, which was similar to that of the 2002 survey. However, we must note that the determination of hypertension in this study was based on the result of the self-report questionnaire, and there may be a part of individuals who were unaware of their hypertension status. In was believed that the rule of halves usually applies to hypertension prevalence in low- and middle-income countries [17]. It
TABLE 3: Inequity analysis of hypertension prevalence by concentration index.

\begin{tabular}{lccccccc}
\hline $\begin{array}{l}\text { Income } \\
\text { group }\end{array}$ & \multicolumn{2}{c}{ Total } & \multicolumn{2}{c}{ Men } & \multicolumn{2}{c}{ Women } & $P$ \\
& Mean & SD & Mean & SD & Mean & SD & value* \\
\hline $\begin{array}{l}\text { Lowest } \\
\text { group }\end{array}$ & 0.28 & 0.448 & 0.25 & 0.436 & 0.30 & 0.457 & 0.722 \\
$\begin{array}{l}\text { Lower group } \\
\text { Medium } \\
\text { group }\end{array}$ & 0.32 & 0.467 & 0.29 & 0.455 & 0.35 & 0.477 & $\mathbf{0 . 0 3 0}$ \\
$\begin{array}{l}\text { Higher } \\
\text { group }\end{array}$ & 0.29 & 0.454 & 0.27 & 0.446 & 0.31 & 0.461 & $\mathbf{0 . 0 0 6}$ \\
$\begin{array}{l}\text { Highest } \\
\text { group }\end{array}$ & 0.29 & 0.456 & 0.28 & 0.448 & 0.31 & 0.462 & 0.083 \\
$\begin{array}{l}\text { Total } \\
\text { CI }\end{array}$ & 0.31 & 0.462 & 0.31 & 0.463 & 0.31 & 0.462 & 0.119 \\
\hline
\end{tabular}

* Significant differences are in bold.

means that half of the hypertension population are aware of their high blood pressure, half of those aware are treated, and half of those treated effectively control the blood pressure. According to this rule, about $60 \%$ of the investigated population may suffer hypertension. However, we should also point out some special conditions to put this result in perspective. With the development of economy and people's living level, more and more people are paying more attention to their body health; periodic physical examination, including blood pressure measurement, is common in Chinese middle-aged and elderly individuals. This must greatly improve the awareness of hypertension. In addition, in this study, $76.5 \%$ people come from rural regions and 43.5\% people are illiterate; this relatively low SES level may also result in increased prevalence of hypertension.

Many demographic and SES factors had been associated with hypertension prevalence. Gender is a usually mentioned influence factor in hypertension. For China, the results of a systematic review in 2014 and a national survey in 2002 both indicated that the prevalence was higher among men than women $[4,17]$. In addition, another research in Chongqing Province of China investigating the prevalence of pre-hypertension also implied a similar result [18]. Our result was consistent with the above, also suggesting that men are more likely to suffer hypertension. Moreover, older age, overweight, and diabetes, which are widely recognized as risk factors to hypertension [19-22], were also found to be associated with hypertension of middle-aged and elderly individuals in China. Among these, overweight and diabetes presented relatively high OR and 95\% CI values, indicating their important roles in the incidence of hypertension among middle-aged and elderly individuals. Considering that the problem of metabolic diseases including obesity and diabetes is increasingly serious in China [23], we should strengthen the emphasis on this issue.

For other SES factors we investigated, poor social support was found to be associated with the incidence of hypertension. In developed countries, lower SES was believed to be associated with higher hypertension prevalence, whereas in undeveloped or developing countries, it was often observed that higher SES was associated with higher blood pressure, and this is usually explained by the opinion 
that in these countries, people with higher SES are also characterized with higher prevalence of obesity, and higher salt and alcohol intakes [13]. At the same time, other researches in developing countries found the same relationship between SES and hypertension prevalence [12]. Our results support the latter opinion, indicating that better SES means lower blood pressure. People with better SES take a new look at their lifestyle and dietary habit, which may explain the phenomenon. In other researches, some other SES factors were also implied to be associated with hypertension. For example, education level, income, and depression had been suggested to be related to hypertension $[19,24,25]$, but negative results were found in this study. Moreover, the calculation of concentration index in different income groups in Table 3 also supports this result, which showed that individuals in different income groups were equally likely to suffer hypertension. Further investigation is needed to explore the role of other SES factors in the prevalence of hypertension, and whether specific country or race may influence the results.

\section{Conclusions}

In summary, in this study, we found that older age, male, overweight, diabetes, and poor society support were associated with the prevalence of hypertension among middleaged and elderly individuals in China. Our study provides implications for better control and management of hypertension.

\section{Data Availability}

The datasets generated during and/or analyzed during the current study are available from the corresponding author on reasonable request.

\section{Conflicts of Interest}

The authors declare that there are no conflicts of interest regarding the publication of this paper.

\section{Supplementary Materials}

Supplementary Table S1: SES factors. (Supplementary Materials)

\section{References}

[1] S. Lewington, B. Lacey, R. Clarke et al., "The burden of hypertension and associated risk for cardiovascular mortality in China," JAMA Internal Medicine, vol. 176, no. 4, pp. 524-532, 2016.

[2] S. Oparil, M. C. Acelajado, G. L. Bakris et al., "Hypertension," Nature Reviews Disease Primers, vol. 4, no. 1, Article ID 18014, 2018.

[3] C. M. Lawes, S. Vander Hoorn, and A. Rodgers, "Global burden of blood-pressure-related disease, 2001," The Lancet, vol. 371, no. 9623, pp. 1513-1518, 2008.

[4] L. Fang, J. Song, Z. Ma, L. Zhang, C. Jing, and D. Chen, "Prevalence and characteristics of hypertension in mainland
Chinese adults over decades: a systematic review," Journal of Human Hypertension, vol. 28, no. 11, pp. 649-656, 2014.

[5] Y. Huang, Z. Deng, Z. Se et al., "Combined impact of risk factors on the subsequent development of hypertension," Journal of Hypertension, vol. 37, no. 4, pp. 696-701, 2019.

[6] P. K. Whelton, R. M. Carey, W. S. Aronow et al., "2017 acc/ aha/aapa/abc/acpm/ags/apha/ash/aspc/nma/pcna guideline for the prevention, detection, evaluation, and management of high blood pressure in adults: executive summary: a report of the american college of cardiology/american heart association task force on clinical practice guidelines," Circulation, vol. 139, no. 25, pp. e426-e483, 2019.

[7] C. Li, L. Dou, H. Wang, S. Jing, and A. Yin, "Horizontal inequity in health care utilization among the middle-aged and elderly in China," International Journal of Environmental Research and Public Health, vol. 14, no. 8, p. 842, 2017.

[8] L. Song, L. Shen, H. Li et al., "Height and prevalence of hypertension in a middle-aged and older Chinese population," Scientific Reports, vol. 6, no. 1, Article ID 39480, 2016.

[9] Y. Zeng, "Toward deeper research and better policy for healthy aging - using the unique data of Chinese longitudinal healthy longevity survey," China Economic Journal, vol. 5, no. 2-3, pp. 131-149, 2012.

[10] E. F. Fang, M. Scheibye-Knudsen, H. J. Jahn et al., "A research agenda for aging in China in the 21st century," Ageing Research Reviews, vol. 24, pp. 197-205, 2015.

[11] J. Liu, S. Rozelle, Q. Xu, N. Yu, and T. Zhou, "Social engagement and elderly health in China: evidence from the China health and retirement longitudinal survey (charls)," International Journal of Environmental Research and Public Health, vol. 16, no. 2, p. 278, 2019.

[12] Z. Yu, A. Nissinen, E. Vartiainen et al., "Associations between socioeconomic status and cardiovascular risk factors in an urban population in China," Bulletin of the World Health Organization, vol. 78, no. 11, pp. 1296-1305, 2000.

[13] H. Colhoun, H. Hemingway, and N. Poulter, "Socio-economic status and blood pressure: an overview analysis," Journal of Human Hypertension, vol. 12, no. 2, pp. 91-110, 1998.

[14] B. Leng, Y. Jin, G. Li, L. Chen, and N. Jin, "Socioeconomic status and hypertension," Journal of Hypertension, vol. 33, no. 2, pp. 221-229, 2015.

[15] P. A. Bath and D. Deeg, "Social engagement and health outcomes among older people: introduction to a special section," European Journal of Ageing, vol. 2, no. 1, pp. 24-30, 2005.

[16] M. Toda, A. Opwora, E. Waweru et al., "Analyzing the equity of public primary care provision in Kenya: variation in facility characteristics by local poverty level," International Journal for Equity in Health, vol. 11, no. 1, p. 75, 2012.

[17] Y. Wu, R. Huxley, L. Li et al., "Prevalence, awareness, treatment, and control of hypertension in China: data from the china national nutrition and health survey 2002," Circulation, vol. 118, no. 25, pp. 2679-2686, 2008.

[18] R. Zhang, R. Deng, P. Shen et al., "Prehypertension and socioeconomic status: a cross-sectional study in chongqing, China," Clinical and experimental hypertension, vol. 39, no. 8 , pp. 774-780, 2017.

[19] E. Y. Tenkorang, V. Kuuire, I. Luginaah, and E. Banchani, "Examining risk factors for hypertension in Ghana: evidence from the study on global ageing and adult health," Global Health Promotion, vol. 24, no. 1, pp. 14-26, 2017. 
[20] D. Prabhakaran, P. Jeemon, S. Ghosh et al., "Prevalence and incidence of hypertension: results from a representative cohort of over 16,000 adults in three cities of south asia," Indian Heart Journal, vol. 69, no. 4, pp. 434-441, 2017.

[21] G. Seravalle and G. Grassi, "Obesity and hypertension," Pharmacological Research, vol. 122, pp. 1-7, 2017.

[22] M. J. Cryer, T. Horani, and D. J. DiPette, "Diabetes and hypertension: a comparative review of current guidelines," The Journal of Clinical Hypertension, vol. 18, no. 2, pp. 95-100, 2016.

[23] J. Lu, L. Wang, M Li et al., "Metabolic syndrome among adults in China: the 2010 China noncommunicable disease surveillance," The Journal of Clinical Endocrinology and Metabolism, vol. 102, pp. 507-515, 2017.

[24] J. Baldisserotto, L. Kopittke, F. B. Nedel et al., "Socio-demographic caracteristics and prevalence of risk factors in a hypertensive and diabetics population: a cross-sectional study in primary health care in Brazil," BMC Public Health, vol. 16, no. 1, p. 573, 2016

[25] S. Sabri, A. Bener, V. Eapen, A. A. Azhar, A. Abdishakure, and J. Singh, "Correlation between hypertension and income distribution among United Arab Emirates population," The Medical Journal of Malaysia, vol. 60, no. 4, pp. 416-425, 2005. 


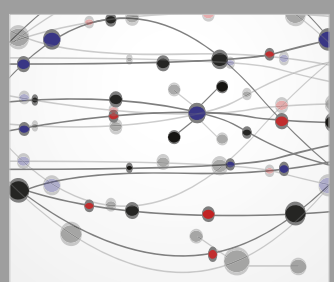

The Scientific World Journal
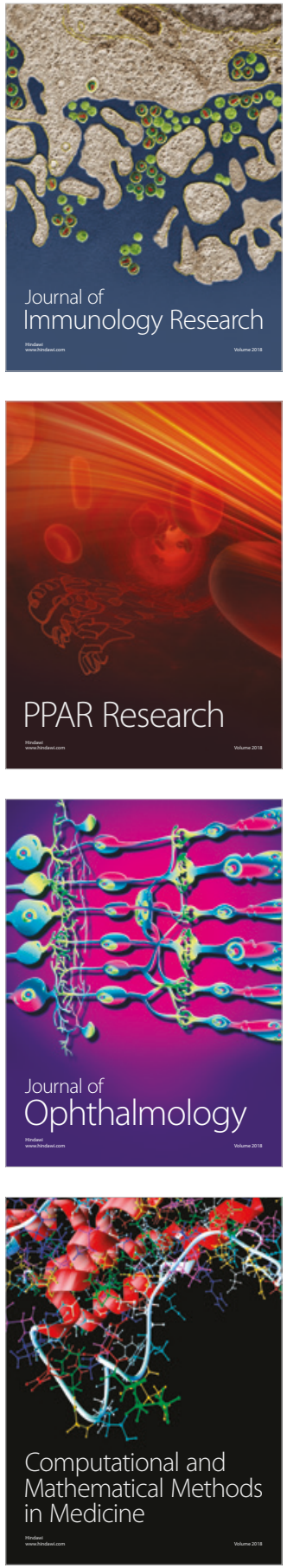

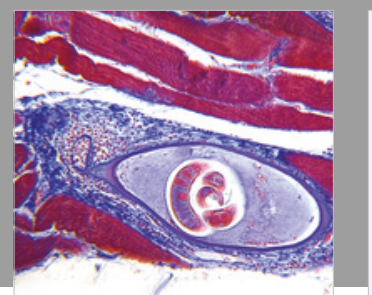

Gastroenterology Research and Practice

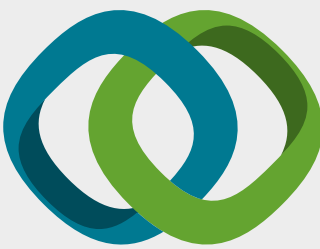

\section{Hindawi}

Submit your manuscripts at

www.hindawi.com
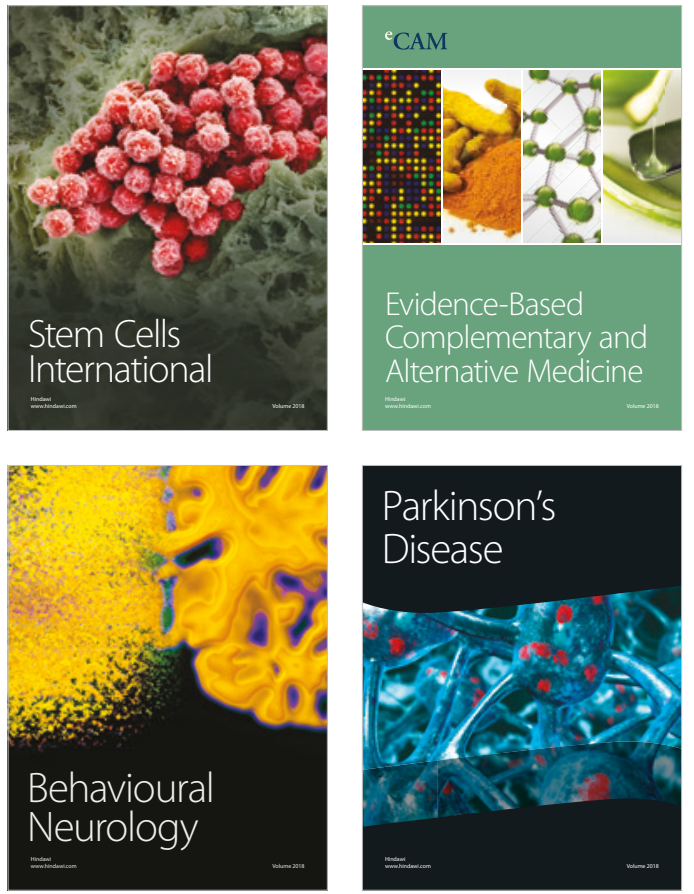

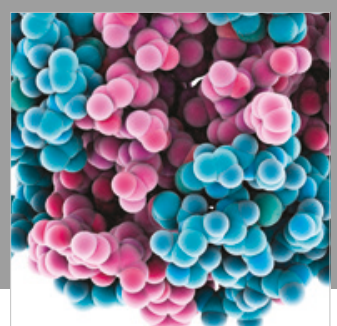

ournal of

Diabetes Research

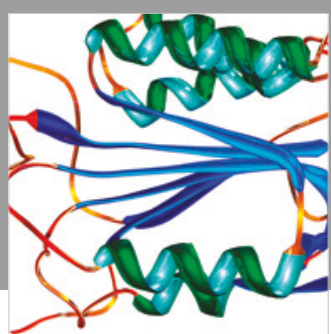

Disease Markers
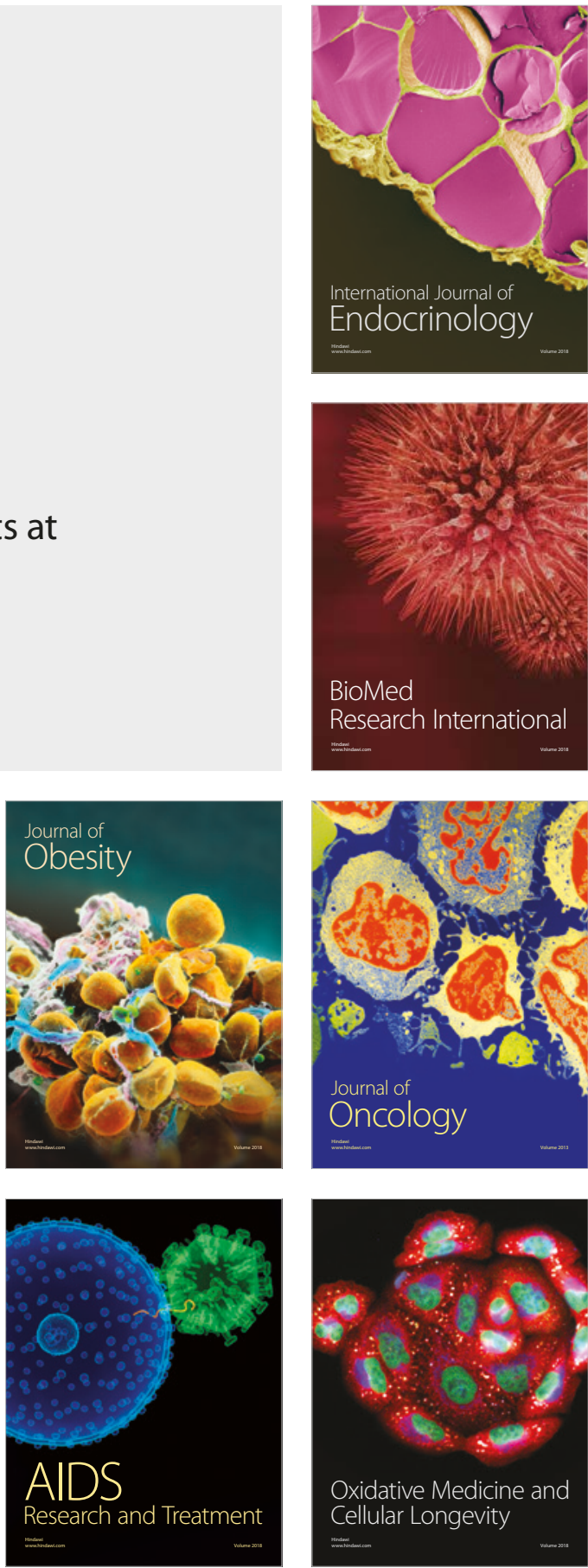